\title{
Implicit Approximation Scheme for the Solution of $K$-Positive Definite Operator Equation
}

\author{
Naseer Shahzad, ${ }^{1}$ Arif Rafiq, ${ }^{2}$ and Habtu Zegeye ${ }^{3}$ \\ ${ }^{1}$ Department of Mathematics, King Abdulaziz University, P.O. Box 80203, Jeddah 21859, Saudi Arabia \\ ${ }^{2}$ Department of Mathematics, Lahore Leads University, Lahore 54810, Pakistan \\ ${ }^{3}$ Departement of Mathematics, University of Botswana, Private Bag Box 00704, Gaborone, Botswana
}

Correspondence should be addressed to Naseer Shahzad; nshahzad@kau.edu.sa

Received 5 December 2013; Accepted 9 February 2014; Published 23 March 2014

Academic Editor: Ljubomir B. Ćirić

Copyright (C) 2014 Naseer Shahzad et al. This is an open access article distributed under the Creative Commons Attribution License, which permits unrestricted use, distribution, and reproduction in any medium, provided the original work is properly cited.

We construct an implicit sequence suitable for the approximation of solutions of $K$-positive definite operator equations in real Banach spaces. Furthermore, implicit error estimate is obtained and the convergence is shown to be faster in comparsion to the explicit error estimate obtained by Osilike and Udomene (2001).

\section{Introduction}

Let $E$ be a real Banach space and let $J$ denote the normalized duality mapping from $E$ to $2^{E^{*}}$ defined by

$$
J(x)=\left\{f^{*} \in E^{*}:\left\langle x, f^{*}\right\rangle=\|x\|^{2},\left\|f^{*}\right\|=\|x\|\right\},
$$

where $E^{*}$ denotes the dual space of $E$ and $\langle\cdot, \cdot\rangle$ denotes the generalized duality pairing. It is well known that if $E^{*}$ is strictly convex, then $J$ is single valued. We will denote the single-valued duality mapping by $j$.

Let $E$ be a Banach space. The modulus of smoothness of $E$ is the function.

$$
\begin{gathered}
\rho_{E}:[0, \infty) \rightarrow[0, \infty) \text { defined by } \\
\rho_{E}(t)=\sup \left\{\frac{1}{2}(\|x+y\|+\|x-y\|)-1:\|x\| \leq 1,\|y\| \leq t\right\} .
\end{gathered}
$$

The Banach space $E$ is called uniformly smooth if

$$
\lim _{t \rightarrow 0} \frac{\rho_{E}(t)}{t}=0 .
$$

A Banach space $E$ is said to be strictly convex if for two elements $x, y \in E$ which are linearly independent we have that $\|x+y\|<\|x\|+\|y\|$.
Let $E_{1}$ be a dense subspace of a Banach space $E$. An operator $T$ with domain $D(T) \supseteq E_{1}$ is called continuously $E_{1}$ invertible if the range of $T, R(T)$, with $T$ in $E$ considered as an operator restricted to $E_{1}$, is dense in $E$ and $T$ has a bounded inverse on $R(T)$.

Let $E$ be a Banach space and let $A$ be a linear unbounded operator defined on a dense domain, $D(A)$, in $E$. An operator $A$ will be called $K$ positive definite $(K p d)$ [1] if there exist a continuously $D(A)$-invertible closed linear operator $K$ with $D(A) \subset D(K)$ and a constant $c>0$ such that $j(K x) \in J(K x)$,

$$
\langle A x, j(K x)\rangle \geq c\|K x\|^{2}, \quad \forall x \in D(A) .
$$

Without loss of generality, we assume that $c \in(0,1)$.

In [1], Chidume and Aneke established the extension of Kpd operators of Martynjuk [2] and Petryshyn [3, 4] from Hilbert spaces to arbitrary real Banach spaces. They proved the following result.

Theorem 1. Let $E$ be a real separable Banach space with a strictly convex dual $E$ and let $A$ be a Kpd operator with $D(A)=$ $D(K)$. Suppose

$$
\langle A x, j(K y)\rangle=\langle K x, j(A y)\rangle, \quad \forall x, y \in D(A) .
$$

Then, there exists a constant $\alpha>0$ such that for all $x \in D(A)$

$$
\|A x\| \leq \alpha\|K x\| .
$$


Furthermore, the operator $A$ is closed, $R(A)=E$, and the equation $A x=f$ has a unique solution for any given $f \in E$.

As the special case of Theorem 1 in which $E=L_{p}\left(l_{p}\right)$ spaces, $2 \leq p<\infty$, Chidume and Aneke [1] introduced an iteration process which converges strongly to the unique solution of the equation $A x=f$, where $A$ and $K$ are commuting. Recently, Chidume and Osilike [5] extended the results of Chidume and Aneke [1] to the more general real separable $q$-uniformly smooth Banach spaces, $1<q<\infty$, by removing the commutativity assumption on $A$ and $K$. Later on, Chuanzhi [6] proved convergence theorems for the iterative approximation of the solution of the Kpd operator equation $A x=f$ in more general separable uniformly smooth Banach spaces.

In [7], Osilike and Udomene proved the following result.

Theorem 2. Let $E$ be a real separable Banach space with a strictly convex dual and let $A: D(A) \subseteq E \rightarrow E$ be a Kpd operator with $D(A)=D(K)$. Suppose $\langle A x, j(K y)\rangle=$ $\langle K x, j(A y)\rangle$ for all $x, y \in D(A)$. Choose any $\epsilon_{1} \in\left(0, c^{2} /(1+\right.$ $\left.\alpha(1-c)+\alpha^{2}\right)$ ) and define $T_{\epsilon}: D(A) \subseteq E \rightarrow E$ by

$$
T_{\epsilon} x=x+\epsilon K^{-1} f-\epsilon K^{-1} A x .
$$

Then the Picard iteration scheme generated from an arbitrary $x_{0} \in D(A)$ by

$$
x_{n+1}=T_{\epsilon} x_{n}=T_{\epsilon}^{n} x_{0}
$$

converges strongly to the solution of the equation $A x=f$. Moreover, if $x^{*}$ denotes the solution of the equation $A x=f$, then

$$
\left\|x_{n+1}-x^{*}\right\| \leq(1-c \epsilon(1-c))^{n} \beta^{-1}\left\|K x_{0}-K x^{*}\right\| .
$$

The most general iterative formula for approximating solutions of nonlinear equation and fixed point of nonlinear mapping is the Mann iterative method [8] which produces a sequence $\left\{x_{n}\right\}$ via the recursive approach $x_{n+1}=\alpha_{n} x_{n}+(1-$ $\left.\alpha_{n}\right) T x_{n}$, for nonlinear mapping $T: C=D(T) \rightarrow C$, where the initial guess $x_{0} \in C$ is chosen arbitrarily. For convergence results of this scheme and related iterative schemes, see, for example, [9-15].

In [16], Xu and Ori introduced the implicit iteration process $\left\{x_{n}\right\}$, which is the modification of Mann, generated by $x_{0} \in C, x_{n}=\alpha_{n} x_{n-1}+\left(1-\alpha_{n}\right) T_{n} x_{n}$, for $T_{i}, i=1,2, \ldots, N$, nonexpansive mappings, and $T_{n}=T_{n}(\bmod N)$ and $\left\{\alpha_{n}\right\} \subset$ $(0,1)$. They proved the weak convergence of this process to a common fixed point of the finite family of nonexpansive mappings in Hilbert spaces. Since then fixed point problems and solving (or approximating) nonlinear equations based on implicit iterative processes have been considered by many authors (see, e.g., [17-21]).

It is our purpose in this paper to introduce implicit scheme which converges strongly to the solution of the Kpd operator equation $A x=f$ in a separable Banach space. Even though our scheme is implicit, the error estimate obtained indicates that the convergence of the implicit scheme is faster in comparison to the explicit scheme obtained by Osilike and Udomene [7].

\section{Main Results}

We need the following results.

Lemma 3 (see [10]). If $E^{*}$ is uniformly convex then there exists a continuous nondecreasing function $b:[0, \infty) \rightarrow[0, \infty)$ such that $b(0)=0, b(\delta t) \leq \delta b(t)$ for all $\delta \geq 1$ and

$$
\|x+y\|^{2} \leq\|x\|^{2}+2\langle y, j(x)\rangle+\max \{\|x\|, 1\}\|y\| b(\|y\|),
$$

for all $x, y \in E$.

Lemma 4 (see [22]). If there exists a positive integer $N$ such that for all $n \geq N, n \in \mathbb{N}$ (the set of all positive integers),

$$
\rho_{n+1} \leq\left(1-\theta_{n}\right) \rho_{n}+b_{n}
$$

then

$$
\lim _{n \rightarrow \infty} \rho_{n}=0
$$

where $\theta_{n} \in[0,1), \sum_{n=1}^{\infty} \theta_{n}=\infty$ and $b_{n}=o\left(\theta_{n}\right)$.

Remark 5 (see [6]). Since $K$ is continuously $D(A)$ invertible, there exists a constant $\beta>0$ such that

$$
\|K x\| \geq \beta\|x\|, \quad \forall x \in D(K)=D(A) .
$$

In the continuation $c \in(0,1), \alpha$ and $\beta$ are the constants appearing in (4), (6), and (12), respectively. Furthermore, $\epsilon>$ 0 is defined by

$$
\epsilon=\frac{c-\eta}{\alpha(1-\eta)}, \quad \eta \in(0, c) .
$$

With these notations, we now prove our main results.

Theorem 6. Let $E$ be a real separable Banach space with a strictly convex dual and let $A: D(A) \subseteq E \rightarrow E$ be a Kpd operator with $D(A)=D(K)$. Suppose $\langle A x, j(K y)\rangle=$ $\langle K x, j(A y)\rangle$ for all $x, y \in D(A)$. Let $x^{*}$ denote a solution of the equation $A x=f$. For arbitrary $x_{0} \in E$, define the sequence $\left\{x_{n}\right\}_{n=0}^{\infty}$ in $E$ by

$$
x_{n}=x_{n-1}+\epsilon K^{-1} f-\epsilon K^{-1} A x_{n}, \quad n \geq 0 .
$$

Then, $\left\{x_{n}\right\}_{n=0}^{\infty}$ converges strongly to $x^{*}$ with

$$
\left\|x_{n}-x^{*}\right\| \leq \rho^{n} \beta^{-1}\left\|K x_{0}-K x^{*}\right\|,
$$

where $\rho=1-((c-\eta) /(\alpha(1-\eta)+c-\eta)) \eta \in(0,1)$. Thus, the choice $\eta=c / 2$ yields $\rho=1-\left(c^{2} /(4 \alpha(1-c / 2)+2 c)\right)$. Moreover, $x^{*}$ is unique.

Proof. The existence of the unique solution to the equation $A x=f$ comes from Theorem 1 . From (4) we have

$$
\langle A x-c K x, j(K x)\rangle \geq 0,
$$

and from Lemma 1.1 of Kato [23], we obtain that

$$
\|K x\| \leq\|K x+\gamma(A x-c K x)\|,
$$


for all $x \in D(A)$ and $\gamma>0$. Now, from (14), linearity of $K$ and the fact that $A x^{*}=f$ we obtain that

$$
\begin{aligned}
K x_{n} & =K x_{n-1}+\epsilon f-\epsilon A x_{n} \\
& =K x_{n-1}+\epsilon A x^{*}-\epsilon A x_{n},
\end{aligned}
$$

which implies that

$$
K x_{n-1}=K x_{n}-\epsilon A x^{*}+\epsilon A x_{n}
$$

so that

$$
K x_{n-1}-K x^{*}=K x_{n}-K x^{*}-\epsilon A x^{*}+\epsilon A x_{n} .
$$

With the help of (14) and Theorem 1, we have the following estimate:

$$
\begin{aligned}
\left\|A x_{n}-A x^{*}\right\| & =\left\|A\left(x_{n}-x^{*}\right)\right\| \leq \alpha\left\|K\left(x_{n}-x^{*}\right)\right\| \\
& =\alpha\left\|K x_{n}-K x^{*}\right\| \\
& =\alpha\left\|K x_{n-1}-K x^{*}-\epsilon\left(A x_{n}-A x^{*}\right)\right\| \\
& \leq \alpha\left\|K x_{n-1}-K x^{*}\right\|+\alpha \epsilon\left\|A x_{n}-A x^{*}\right\|,
\end{aligned}
$$

which gives

$$
\left\|A x_{n}-A x^{*}\right\| \leq \frac{\alpha}{1-\alpha \epsilon}\left\|K x_{n-1}-K x^{*}\right\| .
$$

Furthermore, inequality (20) can be rewritten as

$$
\begin{aligned}
K x_{n-1} & -K x^{*} \\
= & (1+\epsilon)\left(K x_{n}-K x^{*}\right) \\
& +\epsilon\left(A x_{n}-A x^{*}-c\left(K x_{n}-K x^{*}\right)\right) \\
& -\epsilon(1-c)\left(K x_{n}-K x^{*}\right) \\
= & (1+\epsilon)\left[K x_{n}-K x^{*}\right. \\
& \left.\quad+\frac{\epsilon}{1+\epsilon}\left(A x_{n}-A x^{*}-c\left(K x_{n}-K x^{*}\right)\right)\right] \\
& -\epsilon(1-c)\left(K x_{n}-K x^{*}\right) \\
= & (1+\epsilon)\left[K x_{n}-K x^{*}\right. \\
& \left.\quad+\frac{\epsilon}{1+\epsilon}\left(A x_{n}-A x^{*}-c\left(K x_{n}-K x^{*}\right)\right)\right] \\
& -\epsilon(1-c)\left(K x_{n-1}-K x^{*}\right)+\epsilon^{2}(1-c)\left(A x_{n}-A x^{*}\right) .
\end{aligned}
$$

In addition, from (17) and (22), we get that

$$
\begin{aligned}
& \left\|K x_{n-1}-K x^{*}\right\| \\
& \geq(1+\epsilon) \| K x_{n}-K x^{*} \\
& \quad+\frac{\epsilon}{1+\epsilon}\left(A x_{n}-A x^{*}-c\left(K x_{n}-K x^{*}\right)\right) \|
\end{aligned}
$$

$$
\begin{aligned}
& -\epsilon(1-c)\left\|K x_{n-1}-K x^{*}\right\|-\epsilon^{2}(1-c)\left\|A x_{n}-A x^{*}\right\| \\
\geq & (1+\epsilon)\left\|K x_{n}-K x^{*}\right\|-\epsilon(1-c)\left\|K x_{n-1}-K x^{*}\right\| \\
& -\epsilon^{2}(1-c) \frac{\alpha}{1-\alpha \epsilon}\left\|K x_{n-1}-K x^{*}\right\|,
\end{aligned}
$$

which implies that

$$
\begin{aligned}
& \left\|K x_{n}-K x^{* *}\right\| \\
& \quad \leq \frac{1+\epsilon(1-c)+\epsilon^{2}(1-c)(\alpha /(1-\alpha \epsilon))}{1+\epsilon}\left\|K x_{n-1}-K x^{*}\right\| \\
& \quad=\rho\left\|K x_{n-1}-K x^{*}\right\|,
\end{aligned}
$$

where

$$
\begin{aligned}
\rho & =\frac{1+\epsilon(1-c)+\epsilon^{2}(1-c)(\alpha /(1-\alpha \epsilon))}{1+\epsilon} \\
& =1-\frac{\epsilon}{1+\epsilon}\left(c-\epsilon(1-c) \frac{\alpha}{1-\alpha \epsilon}\right) \\
& =1-\frac{\epsilon}{1+\epsilon} \eta \\
& =1-\frac{c-\eta}{\alpha(1-\eta)+c-\eta} \eta \\
& =1-\frac{c^{2}}{4 \alpha(1-c / 2)+2 c} .
\end{aligned}
$$

From (25) and (26), we have that

$$
\left\|K x_{n}-K x^{*}\right\| \leq \rho\left\|K x_{n-1}-K x^{*}\right\| \leq \cdots \leq \rho^{n}\left\|K\left(x_{0}-x^{*}\right)\right\| .
$$

Hence by Remark 5, we get that

$$
\begin{aligned}
& \left\|x_{n}-x^{*}\right\| \\
& \quad \leq \beta^{-1}\left\|K x_{n}-K x^{*}\right\| \leq \cdots \leq \rho^{n} \beta^{-1}\left\|K x_{0}-K x^{*}\right\| \longrightarrow 0,
\end{aligned}
$$

as $n \rightarrow \infty$. Thus, $x_{n} \rightarrow x^{*}$ as $n \rightarrow \infty$.

In [6], Chuanzhi provided the following result.

Theorem 7. Let $E$ be a real uniformly smooth separable Banach space, and let $A: D(A) \subseteq E \rightarrow E$ be a Kpd operator with $D(A)=D(K)$. Suppose $\langle A x, j(K y)\rangle=\langle K x, j(A y)\rangle$ for all $x, y \in D(A)$. For arbitrary $f \in E$ and $x_{0} \in D(A)$, define the sequence $\left\{x_{n}\right\}_{n=0}^{\infty}$ by

$$
\begin{gathered}
x_{n+1}=x_{n}+t_{n} \gamma_{n}, \\
\gamma_{n}=K^{-1} f-K^{-1} A x_{n}, \\
0 \leq t_{n} \leq \frac{1}{2 c}, \\
\sum t_{n}=0, \quad \lim _{n \rightarrow \infty} t_{n}=0, \\
b\left(\alpha t_{n}\right) \leq \frac{2 c}{B \alpha}, \quad n \geq 0,
\end{gathered}
$$


where $b(t)$ is as in $(R), \alpha$ is the constant appearing in inequality (6), $c$ is the constant appearing in inequality (4), and

$$
B=\max \left\{\left\|K \gamma_{0}\right\|, 1\right\} \text {. }
$$

Then, $\left\{x_{n}\right\}_{n=0}^{\infty}$ converges strongly to the unique solution of $A x=$ $f$.

However, its implicit version is as follows.

Theorem 8. Let $E$ be a real uniformly smooth separable Banach space, and let $A: D(A) \subseteq E \rightarrow E$ be a Kpd operator with $D(A)=D(K)$. Suppose $\langle A x, j(K y)\rangle=\langle K x, j(A y)\rangle$ for all $x, y \in D(A)$. For arbitrary $f \in E$ and $x_{0} \in D(A)$, define the sequence $\left\{x_{n}\right\}_{n=0}^{\infty}$ by

$$
\begin{gathered}
x_{n}=x_{n-1}+t_{n} \gamma_{n}, \\
\gamma_{n}=K^{-1} f-K^{-1} A x_{n}, \\
\sum t_{n}=\infty, \quad \lim _{n \rightarrow \infty} t_{n}=0, \quad n \geq 0 .
\end{gathered}
$$

Then, $\left\{x_{n}\right\}_{n=0}^{\infty}$ converges strongly to the unique solution of $A x=$ $f$.

Proof. The existence of the unique solution to the equation $A x=f$ comes from Theorem 1 . Using (31) and (32) we obtain

$$
K \gamma_{n}=K \gamma_{n-1}-t_{n} A \gamma_{n}
$$

Consider

$$
\begin{aligned}
\left\|K \gamma_{n}\right\|^{2} & =\left\langle K \gamma_{n}, j\left(K \gamma_{n}\right)\right\rangle=\left\langle K \gamma_{n-1}-t_{n} A \gamma_{n}, j\left(K \gamma_{n}\right)\right\rangle \\
& =\left\langle K \gamma_{n-1}, j\left(K \gamma_{n}\right)\right\rangle-t_{n}\left\langle A \gamma_{n}, j\left(K \gamma_{n}\right)\right\rangle \\
& \leq\left\|K \gamma_{n-1}\right\|\left\|K \gamma_{n}\right\|-c t_{n}\left\|K \gamma_{n}\right\|^{2},
\end{aligned}
$$

which implies that

$$
\left\|K \gamma_{n}\right\| \leq\left\|K \gamma_{n-1}\right\|-c t_{n}\left\|K \gamma_{n}\right\| .
$$

Hence, $\left\{K \gamma_{n}\right\}_{n=0}^{\infty}$ is bounded. Let

$$
M_{1}=\sup _{n \geq 0}\left\|K \gamma_{n}\right\|
$$

Also from (6) it can be easily seen that $\left\{A \gamma_{n}\right\}_{n=0}^{\infty}$ is also bounded. Let

$$
M_{2}=\sup _{n \geq 0}\left\|A \gamma_{n}\right\| \text {. }
$$

Denote $M=M_{1}+M_{2}$; then $M<\infty$.

By using (34) and Lemma 3, we have

$$
\begin{aligned}
\left\|K \gamma_{n}\right\|^{2}= & \left\|K \gamma_{n-1}-t_{n} A \gamma_{n}\right\|^{2} \\
\leq & \left\|K \gamma_{n-1}\right\|^{2}-2 t_{n}\left\langle A \gamma_{n}, j\left(K \gamma_{n-1}\right)\right\rangle \\
& +\max \left\{\left\|K \gamma_{n-1}\right\|, 1\right\}\left\|t_{n} A \gamma_{n}\right\| b\left(\left\|t_{n} A \gamma_{n}\right\|\right) \\
= & \left\|K \gamma_{n-1}\right\|^{2}-2 t_{n}\left\langle A \gamma_{n-1}, j\left(K \gamma_{n-1}\right)\right\rangle \\
& +2 t_{n}\left\langle A \gamma_{n-1}-A \gamma_{n}, j\left(K \gamma_{n-1}\right)\right\rangle \\
& +\max \left\{\left\|K \gamma_{n-1}\right\|, 1\right\} t_{n}\left\|A \gamma_{n}\right\| b\left(t_{n}\left\|A \gamma_{n}\right\|\right)
\end{aligned}
$$

$$
\begin{aligned}
\leq & \left(1-2 c t_{n}\right)\left\|K \gamma_{n-1}\right\|^{2}+2 t_{n}\left\|A \gamma_{n-1}-A \gamma_{n}\right\|\left\|K \gamma_{n-1}\right\| \\
& +\max \left\{\left\|K \gamma_{n-1}\right\|, 1\right\} \alpha t_{n}\left\|K \gamma_{n}\right\| b\left(\alpha t_{n}\left\|K \gamma_{n}\right\|\right) \\
\leq & \left(1-2 c t_{n}\right)\left\|K \gamma_{n-1}\right\|^{2}+2 M t_{n} \eta_{n} \\
& +\max \{M, 1\} \alpha^{2} M^{2} t_{n} b\left(t_{n}\right),
\end{aligned}
$$

where

$$
\eta_{n}=\left\|A \gamma_{n-1}-A \gamma_{n}\right\| \text {. }
$$

By using (6) and (34) we obtain that

$$
\begin{aligned}
\left\|A \gamma_{n-1}-A \gamma_{n}\right\| & =\left\|A\left(\gamma_{n-1}-\gamma_{n}\right)\right\| \leq \alpha\left\|K\left(\gamma_{n-1}-\gamma_{n}\right)\right\| \\
& =\alpha t_{n}\left\|A \gamma_{n}\right\| \leq M \alpha t_{n} \longrightarrow 0, \quad \text { as } n \longrightarrow \infty .
\end{aligned}
$$

Thus,

$$
\eta_{n} \longrightarrow 0 \quad \text { as } n \longrightarrow \infty
$$

Denote

$$
\begin{gathered}
\rho_{n}=\left\|x_{n}-p\right\|, \\
\theta_{n}=2 c t_{n}, \\
\sigma_{n}=2 M t_{n} \eta_{n}+\max \{M, 1\} \alpha^{2} M^{2} t_{n} b\left(t_{n}\right) .
\end{gathered}
$$

Condition (33) assures the existence of a rank $n_{0} \in \mathbb{N}$ such that $\theta_{n}=2 c t_{n} \leq 1$, for all $n \geq n_{0}$. Since $b(t)$ is continuous, so $\lim _{n \rightarrow \infty} b\left(t_{n}\right)=0$ (by condition (33)). Now with the help of (33), (42), and Lemma 4, we obtain from (39) that

$$
\lim _{n \rightarrow \infty}\left\|K \gamma_{n}\right\|=0 \text {. }
$$

At last by Remark 5, $\gamma_{n} \rightarrow 0$ as $n \rightarrow \infty$; that is $A x_{n} \rightarrow f$ as $n \rightarrow \infty$. Because $A$ has bounded inverse, this implies that $x_{n} \rightarrow A^{-1} f$, the unique solution of $A x_{n}=f$. This completes the proof.

Remark 9. (1) According to the estimates (6-8) of Martynjuk [2], we have

$$
\begin{aligned}
& \left\|K x_{n+1}-K x^{*}\right\| \\
& \quad \leq \frac{1+\epsilon_{1}(1-c)+\alpha \epsilon_{1}^{2}(1-c+\alpha)}{1+\epsilon_{1}}\left\|K x_{n}-K x^{*}\right\| \\
& \quad=\theta\left\|K x_{n}-K x^{*}\right\|,
\end{aligned}
$$

where

$$
\begin{aligned}
\theta & =\frac{1+\epsilon_{1}(1-c)+\alpha \epsilon_{1}^{2}(1-c+\alpha)}{1+\epsilon_{1}} \\
& =1-\frac{\epsilon_{1}}{1+\epsilon_{1}}\left(c-\alpha(1-c+\alpha) \epsilon_{1}\right) \\
& =1-\frac{\epsilon_{1}}{1+\epsilon_{1}} \eta,
\end{aligned}
$$

for $\eta=c-\alpha(1-c+\alpha) \epsilon_{1}$ or $\epsilon_{1}=(c-\eta) / \alpha(1-c+\alpha), \eta \in(0, c)$. Thus,

$$
\begin{aligned}
\theta & =1-\frac{c-\eta}{\alpha(1-c+\alpha)+c-\eta} \eta \\
& =1-\frac{c^{2}}{4 \alpha(1-c+\alpha)+2 c} .
\end{aligned}
$$


TABle 1

\begin{tabular}{ccccccccc}
\hline$n$ & 1 & 2 & 3 & 4 & 5 & 6 & 7 & 8 \\
\hline$x_{n}$ & 0.0922 & 0.0851 & 0.07859 & 0.07528 & 0.06947 & 0.06411 & 0.05916 & 0.05459 \\
\hline
\end{tabular}

TABLE 2

\begin{tabular}{ccccccccc}
\hline$n$ & 1 & 2 & 3 & 4 & 5 & 6 & 7 & 8 \\
\hline$x_{n}$ & 0.0893 & 0.0798 & 0.07136 & 0.06376 & 0.05698 & 0.05092 & 0.04550 & 0.04066 \\
\hline
\end{tabular}

TABle 3

\begin{tabular}{ccccccccc}
\hline$n$ & 1 & 2 & 3 & 4 & 5 & 6 & 7 & 8 \\
\hline$x_{n}$ & 0.0098 & 0.0096 & 0.00949 & 0.00933 & 0.00917 & 0.00901 & 0.00885 & 0.00870 \\
\hline
\end{tabular}

TABle 4

\begin{tabular}{ccccccccc}
\hline$n$ & 1 & 2 & 3 & 4 & 5 & 6 & 7 & 8 \\
\hline$x_{n}$ & 0.0098 & 0.0096 & 0.00941 & 0.00923 & 0.00905 & 0.00887 & 0.00869 & 0.00852 \\
\hline
\end{tabular}

(2) For $\alpha>c / 2$, we observe that

$$
\begin{aligned}
\rho & =1-\frac{c^{2}}{4 \alpha(1-c / 2)+2 c} \\
& =\theta-\frac{4 \alpha c^{2}}{(4 \alpha(1-c / 2)+2 c)(4 \alpha(1-c+\alpha)+2 c)}\left(\alpha-\frac{c}{2}\right) .
\end{aligned}
$$

Thus, the relation between Martynjuk [2] and our parameter of convergence, that is, between $\theta$ and $\rho$, respectively, is the following:

$$
\rho<\theta
$$

Despite the fact that our scheme is implicit, inequality (49) shows that the results of Osilike and Udomene [7] are improved in the sense that our scheme converges faster.

Example 10. Suppose $E=\mathbb{R}, D(A)=\mathbb{R}_{+}, A x=x, K x=$ $2 I\left(x^{*}=0\right.$ is the solution of $\left.A x=f\right)$; then for the explicit iterative scheme due to Osilike and Udomene [7] we have

$$
K x_{n+1}=K x_{n}-\epsilon_{1} A x_{n}
$$

which implies that

$$
2 x_{n+1}=2 x_{n}-\epsilon_{1} x_{n},
$$

and hence

$$
x_{n+1}=\left(1-\frac{\epsilon_{1}}{2}\right) x_{n} .
$$

Also for the implicit iterative scheme we have that

$$
K x_{n}=K x_{n-1}-\epsilon A x_{n},
$$

which implies that

$$
x_{n}=\frac{1}{1+\epsilon / 2} x_{n-1} .
$$

It can be easily seen that for $c \leq 1 / 2$ and $\alpha \geq 1 / 2$, (4) and (6) are satisfied. Suppose $c=1 / 4$ and $\alpha=3 / 5$; then $\eta=0.125$, $\epsilon=(c-\eta) / \alpha(1-\eta)=0.23810, \epsilon_{1}=(c-\eta) / \alpha(1-c+\alpha)=$ $0.15432, \rho=0.97596$, and $\theta=0.983288$ and so $\rho<\theta$. Take $x_{0}=0.1$; then from (52) we have Table 1 and for (54) we get Table 2.

Example 11. Let us take $E=\mathbb{R}, D(A)=\mathbb{R}_{+}, A x=(1 / 4) x$, $K x=2 x\left(x^{*}=0\right.$ is the solution of $\left.A x=f\right)$; then for the explicit iterative scheme due to Osilike and Udomene [7] we have

$$
K x_{n+1}=K x_{n}-\epsilon_{1} A x_{n},
$$

which implies that

$$
2 x_{n+1}=2 x_{n}-\frac{\epsilon_{1}}{4} x_{n},
$$

and hence

$$
x_{n+1}=\left(1-\frac{\epsilon_{1}}{8}\right) x_{n} .
$$

Also for the implicit iterative scheme we have that

$$
K x_{n}=K x_{n-1}-\epsilon A x_{n},
$$

which implies that

$$
x_{n}=\frac{1}{1+\epsilon / 8} x_{n-1} .
$$

It can be easily seen that for $c \leq 1 / 8$ and $\alpha \geq 1 / 8$, (4) and (6) are satisfied. Suppose $c=0.0625$ and $\alpha=0.2$; then $\eta=$ $0.03125, \epsilon=(c-\eta) / \alpha(1-\eta)=0.16129, \epsilon_{1}=(c-\eta) / \alpha(1-$ $c+\alpha)=0.13736, \rho=0.99566$, and $\theta=0.99623$ and so $\rho<\theta$. Take $x_{0}=0.01$; then from (57) we have Table 3 and for (59) we get Table 4 .

Even though our scheme is implicit we observe that it converges strongly to the solution of the Kpd operator equation $A x=f$ with the error estimate which is faster in comparison to the explicit error estimate obtained by Osilike and Udomene [7]. 


\section{Conflict of Interests}

The authors declare that there is no conflict of interests regarding the publication of this paper.

\section{Acknowledgments}

This article was funded by the Deanship of Scientific Research (DSR), King Abdulaziz University, Jeddah. The first author, therefore, acknowledges his thanks to DSR for the financial support. This paper is dedicated to Professor Miodrag Mateljevi'c on the occasion of his 65th birthday.

\section{References}

[1] C. E. Chidume and S. J. Aneke, "Existence, uniqueness and approximation of a solution for a $K$-positive definite operator equation," Applicable Analysis, vol. 50, no. 3-4, pp. 285-294, 1993.

[2] A. E. Martynjuk, "Some new applications of methods of Galerkin type," vol. 49, no. 1, pp. 85-108, 1959.

[3] W. V. Petryshyn, "Direct and iterative methods for the solution of linear operator equations in Hilbert space," Transactions of the American Mathematical Society, vol. 105, no. 1, pp. 136-175, 1962.

[4] W. V. Petryshyn, "On a class of $K$-p.d. and non- $K$-p.d. operators and operator equations," Journal of Mathematical Analysis and Applications, vol. 10, no. 1, pp. 1-24, 1965.

[5] C. E. Chidume and M. O. Osilike, "Approximation of a solution for a K-Positive definite operator equation," Journal of Mathematical Analysis and Applications, vol. 210, no. 1, pp. 1-7, 1997.

[6] B. Chuanzhi, "Approximation of a solution for a $K$-positive definite operator equation in uniformly smooth separable Banach spaces," Journal of Mathematical Analysis and Applications, vol. 236, no. 2, pp. 236-242, 1999.

[7] M. O. Osilike and A. Udomene, "A note on approximation of solutions of a $K$-positive definite operator equations," Bulletin of the Korean Mathematical Society, vol. 38, no. 2, pp. 231-236, 2001.

[8] W. R. Mann, "Mean value methods in iteration," Proceedings of the American Mathematical Society, vol. 4, pp. 506-510, 1953.

[9] Z. Opial, "Weak convergence of the sequence of successive approximations for nonexpansive mappings," Bulletin of the American Mathematical Society, vol. 73, pp. 591-597, 1967.

[10] S. Reich, "An iterative procedure for constructing zeros of accretive sets in Banach spaces," Nonlinear Analysis: Theory, Methods \& Applications, vol. 2, no. 1, pp. 85-92, 1978.

[11] N. Shahzad and H. Zegeye, "On Mann and Ishikawa iteration schemes for multi-valued maps in Banach spaces," Nonlinear Analysis: Theory, Methods \& Applications, vol. 71, no. 3-4, pp. 838-844, 2009.

[12] C. H. Xiang, Z. Chen, and K. Q. Zhao, "Some convergence theorems for a class of generalized $\Phi$-hemicontractive mappings," Journal of Concrete and Applicable Mathematics, vol. 8, no. 4, pp. 638-644, 2010

[13] Y. Xu, "Ishikawa and Mann iterative processes with errors for nonlinear strongly accretive operator equations," Journal of Mathematical Analysis and Applications, vol. 224, no. 1, pp. 91101, 1998.

[14] H. Zegeye and N. Shahzad, “Convergence of Mann's type iteration method for generalized asymptotically nonexpansive mappings," Computers and Mathematics with Applications, vol. 62, no. 11, pp. 4007-4014, 2011.

[15] H. Zegeye and N. Shahzad, "Approximation of the common minimum-norm fixed point of a finite family of asymptotically nonexpansive mappings," Fixed Point Theory and Applications, vol. 2013, no. 1, article 1, 2013.

[16] H.-K. Xu and R. G. Ori, "An implicit iteration process for nonexpansive mappings," Numerical Functional Analysis and Optimization, vol. 22, no. 5-6, pp. 767-773, 2001.

[17] N. Shahzad and H. Zegeye, "Strong convergence of an implicit iteration process for a finite family of generalized asymptotically quasi-nonexpansive maps," Applied Mathematics and Computation, vol. 189, no. 2, pp. 1058-1065, 2007.

[18] N. Shahzad and H. Zegeye, "Strong convergence results for nonself multimaps in Banach spaces," Proceedings of the American Mathematical Society, vol. 136, no. 2, pp. 539-548, 2008.

[19] Y. Zhou and S.-S. Chang, "Convergence of implicit iteration process for a finite family of asymptotically nonexpansive mappings in Banach spaces," Numerical Functional Analysis and Optimization, vol. 23, no. 7-8, pp. 911-921, 2002.

[20] N. Shahzad and H. Zegeye, "Viscosity approximation methods for nonexpansive multimaps in Banach spaces," Acta Mathematica Sinica, vol. 26, no. 6, pp. 1165-1176, 2010.

[21] C. E. Chidume and N. Shahzad, "Strong convergence of an implicit iteration process for a finite family of nonexpansive mappings," Nonlinear Analysis: Theory, Methods \& Applications, vol. 62, no. 6, pp. 1149-1156, 2005.

[22] X. Weng, "Fixed point iteration for local strictly pseudocontractive mapping," Proceedings of the American Mathematical Society, vol. 113, no. 3, pp. 727-731, 1991.

[23] T. Kato, "Nonlinear semigroups and evolution equations," Journal of the Mathematical Society of Japan, vol. 19, no. 4, pp. 508-520, 1967. 


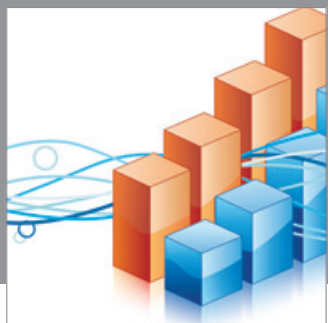

Advances in

Operations Research

mansans

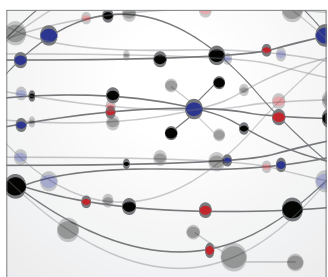

The Scientific World Journal
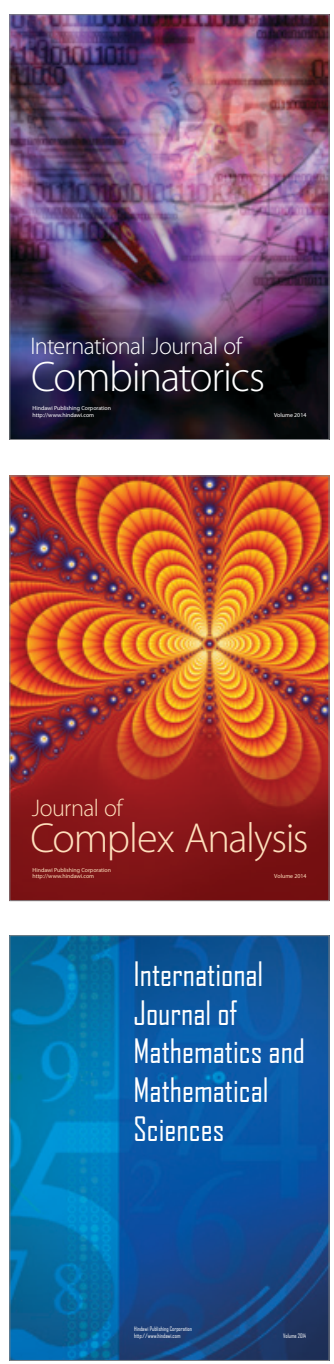
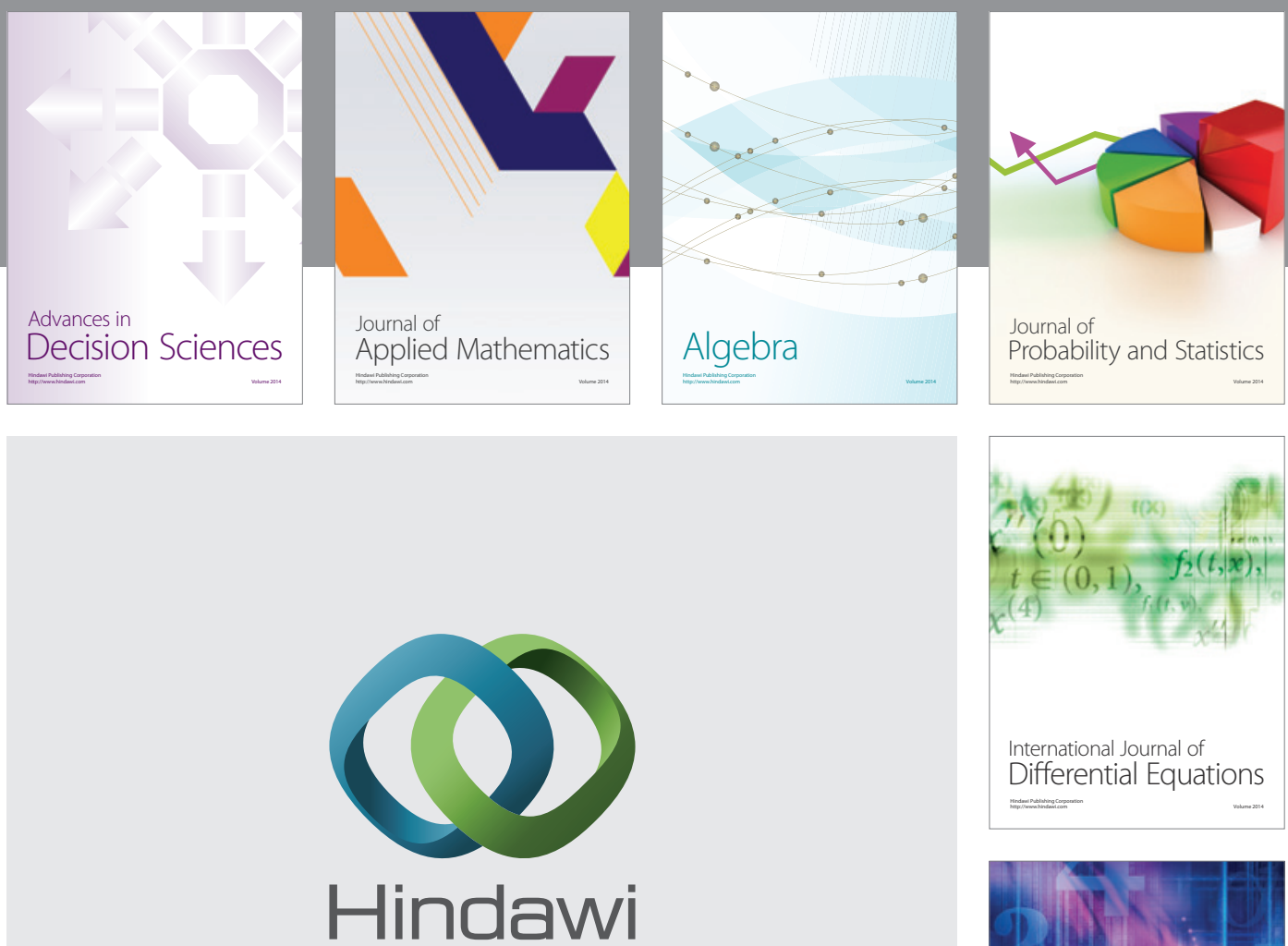

Submit your manuscripts at http://www.hindawi.com
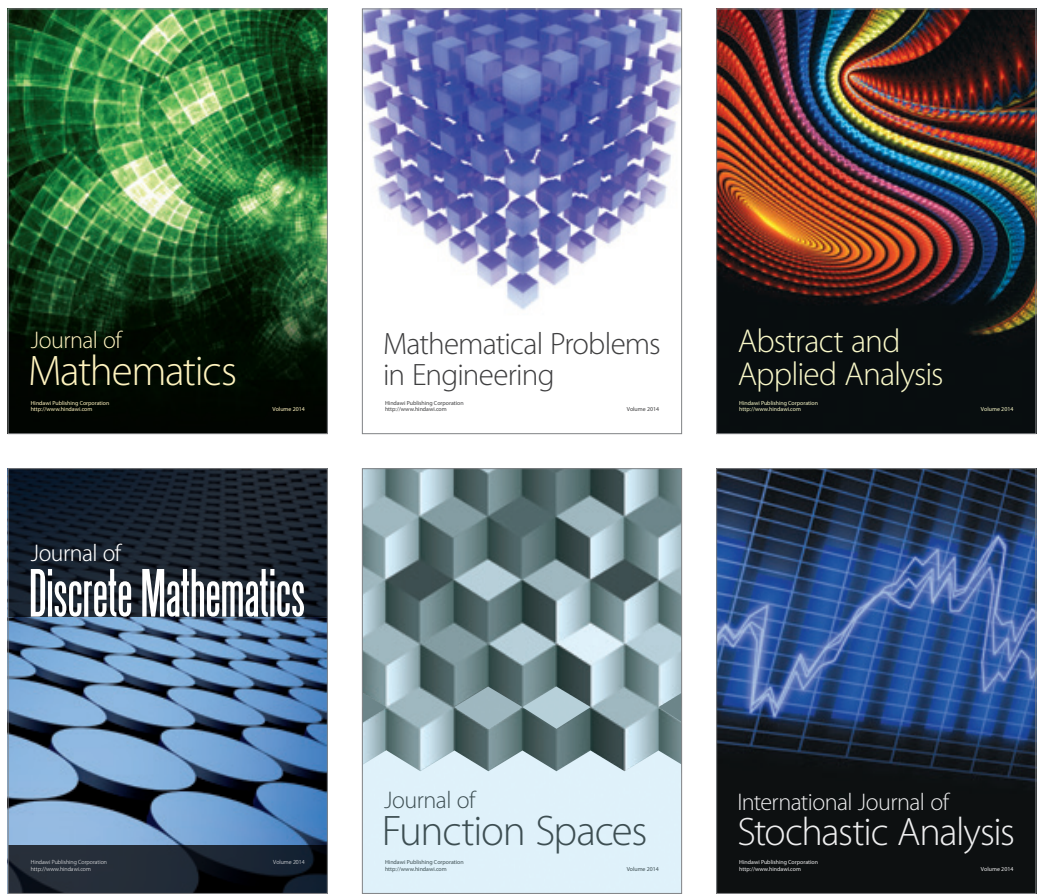

Journal of

Function Spaces

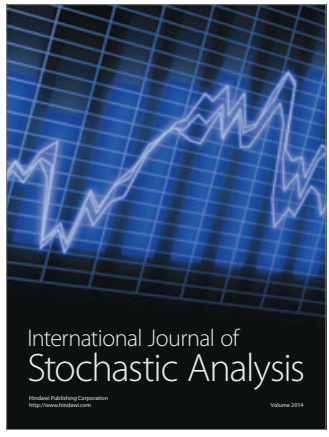

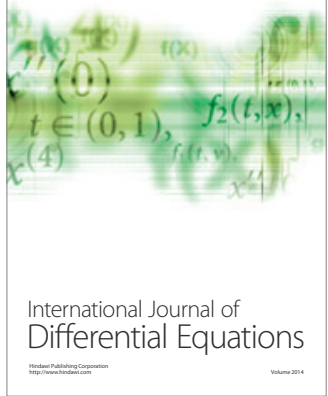
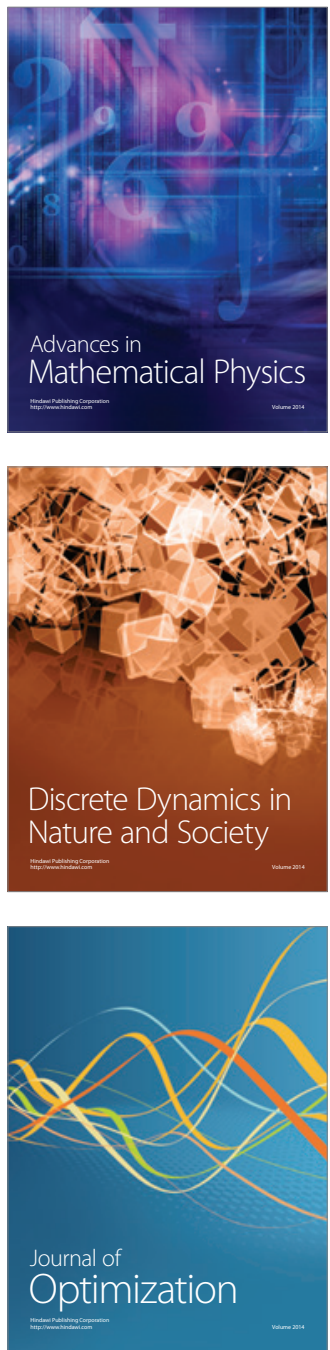